\title{
MEMORIES, TRAJECTORIES AND BEREAVEMENT
}

Penny Mills, Frances Griffiths, Dan Munday Warwick Medical School, University of Warwick, Coventry England

Introduction Much attention has been given in recent years to the concept of illness trajectories and their potential use in the planning of services provided to those with life limiting illnesses. This study examines how trajectories are experienced by bereaved individuals and whether the memories retained by the bereaved or the experience of grief differ according to the disease trajectory of the deceased.

Aims and methods The aim is to investigate whether the trajectory of dying influences the experience of grief or the memories held by the bereaved of the death and prior biography of the deceased.

One to one unstructured in depth interviews have been conducted with bereaved relatives between 3 months and 2 years after the death, followed by thematic analysis of the narratives produced.

Preliminary results While it is possible to identify key stages in the dying trajectory as experienced by the bereaved, there is little uniformity to trajectories and key events are often not related to the medical status of the deceased.

Considerable sacrifices are often made by the bereaved in order to achieve a'good death' as defined by the deceased and there appears to be a need to integrate the trajectory of dying with the memories of the deceased and their prior biography.

Discussion There are a wide variety of experiences, with differences between the medical and the experienced trajectories of dying.

Research is ongoing, but it seems that the needs of the bereaved can be (willingly) overridden in the desire to provide an appropriate death and retain coherent memories of the deceased.

This may have future implications for the assessment of need and the provision of services and support of the bereaved both prior to and following the death. 